\title{
Numerical investigation of critical states in superposed superconducting films
}

\author{
L. Burger*, I. S. Veshchunov ${ }^{\dagger}$, T. Tamegai ${ }^{\dagger}$, A. V. Silhanek ${ }^{\ddagger}$, S. Nagasawa ${ }^{\S}$, M. Hidaka ${ }^{\S}$ and B. Vanderheyden* \\ ${ }^{*}$ SUPRATECS group, Montefiore Research Unit, Department of Electrical Engineering and Computer Science, Université de \\ Liège, 4000 Sart Tilman, Belgium \\ ${ }^{\dagger}$ Department of Applied Physics, The University of Tokyo, Hongo, Tokyo, Japan \\ $\ddagger$ Experimental Physics of Nanostructured Materials, Q-MAT, CESAM, Université de Liège, 4000 Sart Tilman, Belgium

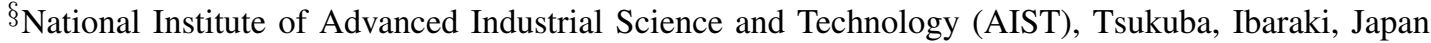 \\ E-mail: lburger@uliege.be
}

\begin{abstract}
This paper examines the magnetic field and supercurrent distributions in three-dimensional structures made of superposed superconducting films. A recent experimental study of such structures (Tamegai 2017) has shown unusual patterns of the trapped magnetic flux, with an unexpected discontinuity line in the region where the films overlap. In this work, a finite element model is used to reproduce and understand these patterns. It is shown that the newly observed discontinuity line originates from the magnetic field dependence of the critical current density. Moreover, in contrast to common observations made in planar superconducting structures, the network of critical lines is found to evolve with the applied field when the three-dimensional structure is magnetized after zero-field cooling. These findings underline the importance of the magnetic coupling between the components of a multi-layered superconducting structure in building its critical state.
\end{abstract}

Index Terms-FEM, critical state, coupling, superconductivity, thin films.

\section{INTRODUCTION}

Over the last decade, progresses in top-down nanofabrication techniques has lead to a wide development of superconducting planar structures with well-mastered electrical and magnetic properties [1]. Nowadays, the fabrication of three-dimensional (3D) superconducting structures offers new possibilities for developing superconducting devices and maximizing their functionalities. 3D integration techniques have recently been identified as promising routes for large-scale superconducting electronic systems [2]. Several superconducting sensors already integrate inherently 3D structures, such as step-edge Josephson junctions [3] or SQUID devices coupled to a freestanding pickup loop made of tungsten nanowires that probe in-plane magnetic fields [4]. Complex multi-layered arrangements also enable the design of superconducting metamaterials with novel functionalities, such as magnetic flux concentration [5], which may increase the sensitivity of magnetic sensors, or magnetic cloaking [6], enabling the attenuation of the magnetic flux density in a given region with no disturbance to the environment. Moreover, 3D structures have proven to be beneficial in large-scale systems, for instance in twisted Roebel cables, that profit from reduced transport AC losses [7], and in magnetized stacks of tapes, that may be used as powerful permanent magnets in superconducting motors [8], [9], [10].

While offering enhanced or new functionalities, 3D superconducting structures have a richer magnetic response than planar devices. A particular class of 3D structures is obtained by stacking nanostructured superconducting layers, resulting in systems where the planar subcomponents are magnetically coupled with one another, thereby opening the possibilities of new magnetic flux penetration processes. Magneto-optical studies have for instance shown that arrays of stacked superconducting strips may sustain thermomagnetic instabilities in the form of anomalous linear avalanches traversing the different layers, provided their overlap is large [11]. More recently, magneto-optical imaging of bi-layer systems showed critical states that cannot be accounted for from a simple combination of the critical states obtained in each layer separately [12].

Critical states in planar superconducting structures are characterized by discontinuity lines (d-lines) where the current density changes abruptly its direction. Such lines are easily identified in magneto-optical images, since the large demagnetizing effects of thin films give rise to a sharp variation of the magnetic field strength around d-lines and hence a high contrast variation. D-lines typically appear along symmetry axes and form a pattern intimately related to the geometry of the film and its current-carrying properties [13], [14]. For instance, in square films, d-lines appear along diagonals (thus forming an " $\mathrm{X}$ " pattern) and delimit a $\pi / 2$ angular change in the direction of the current density, as shown in Fig. 1a. In rectangular films, d-lines run along the bisector of each corner angle and merge into a straight segment extending in the middle of the film, forming a "double-Y" shape, as represented in Fig. 1b. D-lines are also generated by defects and extend over distances much larger than the defect characteristic length [15], [16]. They are further affected by the presence of holes [17], or an anisotropy in the critical current density $j_{c}$ [18], [19], as their angle needs to be consistent with the continuity of the current crossing them [13], [14]. In all these cases, the critical state is determined once and for all from the geometrical and critical current density distribution in the sample. More specifically, for planar structures, the shape of the d-lines does not change with the applied field.

Recently, a new type of pattern was experimentally identified by the group of Tamegai [12] in a 3D structure made of a superconducting strip on top of a square superconducting film (the strip and the square were separated by an electrically insulating $\mathrm{SiO}_{2}$ layer, as schematically depicted in Fig. 1d). As observed in the magneto-optical image of the sample at the remanent state (Fig. 1c), the magnetic field pattern 


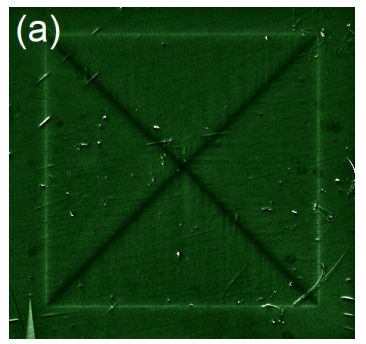

(b)
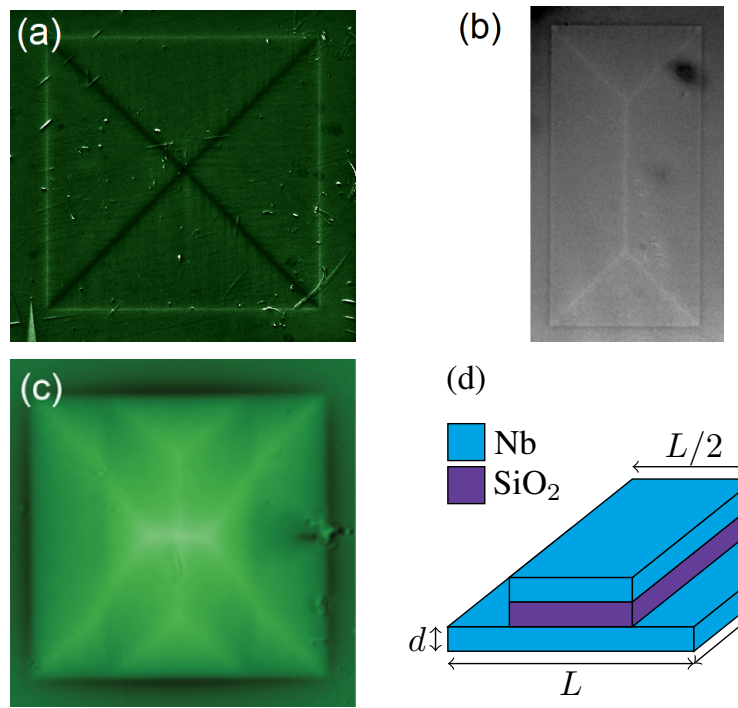

(d)

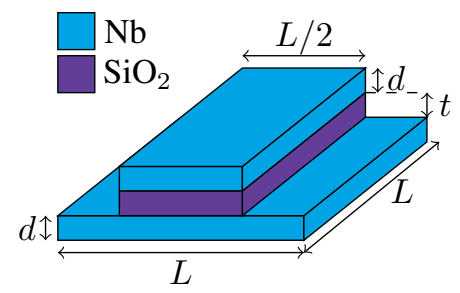

Fig. 1. Magneto-optical image of the critical state in a completely penetrated square superconducting film (a), in a rectangular superconducting strip in the remanent state(b), and in the superposition of a rectangular superconducting strip over a square superconducting film in the remanent state (c). The geometry of the assembly corresponding to the image in panel (c) is depicted in (d). For every magneto-optical image, the brighter the contrast, the higher the magnetic field.

shows an unexpected d-line extending around the center of the structure, which is perpendicular to the long edge of the strip, and is absent from the critical state of the square film alone or that of the strip alone. The purpose of this work is to shed light on the origin of this new critical state structure. To that end, we perform numerical simulations for the penetration of magnetic flux in the square+strip assembly after a zero-field cooling procedure. Our simulations show that d-lines patterns reproduce the expected trend observed in planar structures during the early stages of penetration. However, the magnetic coupling in the region of overlap is found to lead to (1) a structure which varies with the applied field in the subsequent stages of magnetization, and (2) the horizontal line in the remanent state which was experimentally observed in Ref. [12].

The studied samples and experimental methods are presented in Sec. II, while the numerical model is described in Sec. III. Simulation results are presented in Sec IV and discussed in Sec. V, where the crucial role played by magnetic field dependence of the critical current density is emphasized and a simplified model is suggested. Conclusions are drawn in Sec. VI.

\section{EXPERIMENTS}

Bi-layered superconducting $\mathrm{Nb}$ films were fabricated on $\mathrm{Si}$ substrates by using magnetron sputtering, photolithography, and $\mathrm{SF}_{6}$ reactive ion etching technique. To make a reliable top strip on top of the bottom square film, caldera planarization was applied [20]. Two $300 \mathrm{~nm}$ thick $\mathrm{Nb}$ films were separated by a $300 \mathrm{~nm}$ thick $\mathrm{SiO}_{2}$ layer. Flux penetrations and critical state field profiles into the bi-layered $\mathrm{Nb}$ films were captured by magneto-optical imaging technique (MOI), where spatial variation of out-of-plane flux density is detected using the

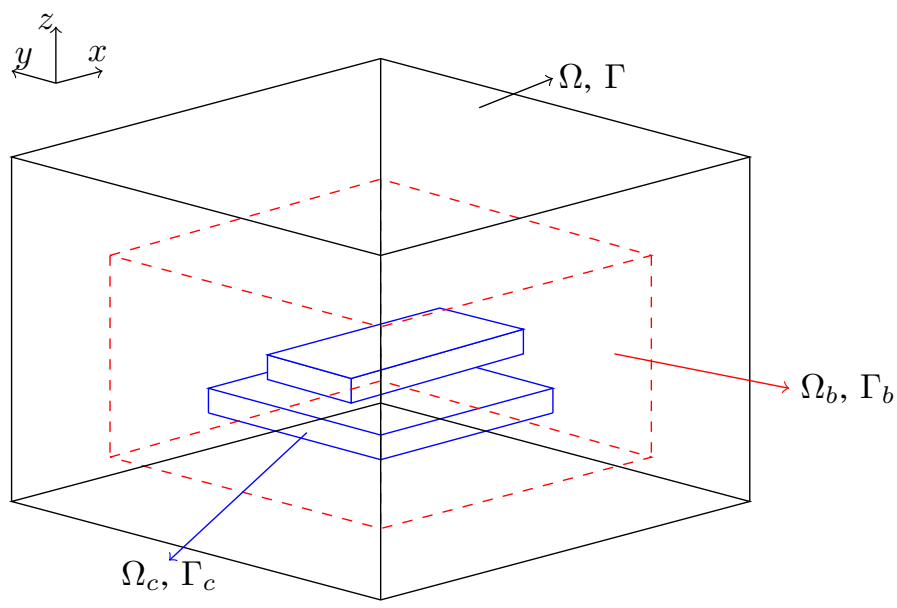

Fig. 2. Domains considered in the $H-\phi$ formulation. $\Omega$ is the computational domain, $\Omega_{c}$ contains the superconducting materials, and $\Omega_{b}$ is an auxiliary box introduced for the application of the external field. The corresponding boundaries of the domains are $\Gamma, \Gamma_{c}$, and $\Gamma_{b}$ respectively.

Faraday effect in a ferromagnetic garnet film in direct contact with the sample [11], [12], [21]. We used a commercial optical microscope (Olympus BX30MF) and a cooled-CCD camera with 12-bit resolution (ORCA-ER, Hamamatsu) to capture MO images. Samples are cooled using a He-flow cryostat (Microstat HighRes II, Oxford Instruments) down to $6 \mathrm{~K}$ under zero applied magnetic field. The magnetooptically determined critical temperature of the $\mathrm{Nb}$ samples was $T_{c}=9 \mathrm{~K}$, consistent with the onset of diamagnetism measured by SQUID magnetometer. A magnetic field was applied perpendicular to the film plane. In order to remove inplane magnetic domains in the ferromagnetic magneto-optical indicator, permanent magnets were used to generate a $180 \mathrm{Oe}$ in-plane magnetic field, parallel to a diagonal of the $\mathrm{Nb}$ square film.

\section{NUMERICAL MODEL}

In order to model the penetration of magnetic flux inside the assembly, we use a finite-element method with a threedimensional $H-\phi$ formulation [22]. The magnetic field is expressed as $\vec{H}=\vec{H}_{a}+\vec{h}$, where $\vec{H}_{a}$ is the applied field and $\vec{h}$ is the reaction field arising from the superconducting currents. Faraday's law is solved in the weak form,

$$
\begin{aligned}
& \int_{\Omega} \mu_{0} \dot{\vec{H}}_{a} \cdot \vec{\psi} d \Omega+\int_{\Omega} \mu_{0} \dot{\vec{h}} \cdot \vec{\psi} d \Omega \\
+ & \int_{\Omega_{c}} \rho(|\vec{\nabla} \times \vec{h}|)(\vec{\nabla} \times \vec{h}) \cdot(\vec{\nabla} \times \vec{\psi}) d \Omega=0,
\end{aligned}
$$

where $\mu_{0}$ is the vacuum permeability, $\rho$ is the electrical resistivity in the superconducting regions, $\dot{\vec{H}}_{a}$ is the rate of variation of the applied field, and $\vec{\psi}$ are the finite element test functions. Here, $\Omega$ is the total computation domain while $\Omega_{c}$ contains the superconducting materials, see Fig. 2. In $\Omega_{c}, \vec{\psi}$ are first-order curl-conforming elements. In $\Omega / \Omega_{c}$, as this set is simply connected, the test functions $\vec{\psi}$ can be written as $\vec{\psi}=-\vec{\nabla} \varphi$, where $\varphi$ is represented with nodal functions. 
The implementation of the method includes two further building blocks that are directly related to the flat geometry of the system [22]. First, boundary conditions at infinite distances are applied by means of a unidirectional shelltransformation. Denoting as $\hat{z}$ the direction perpendicular to the superconducting films, the change of variable

$$
z=f(Z)=B-\frac{A(B-A)}{Z}
$$

maps the infinitely extended physical domain $\Omega_{p h}$, of coordinates $Z$, into a shell domain $\Omega_{s h}$ of finite extension and coordinates $z$, as illustrated in Fig. 3. This transformation allows the calculations to be carried out in a finite-size computational domain without any truncation approximation. Moreover, the $1 / Z^{2}$ asymptotic decay of the magnetic potential can be accurately captured with a suitable choice of the parameters $A$ and $B$. The second building block deals with the application of a uniform field $\vec{H}_{a}$ to the superconducting system. An exploitation of the divergence-free property of $\vec{H}_{a}$ allows us to rewrite Eq. (1) in such a way that only elements in an auxiliary box $\Omega_{b}$ and its boundary $\Gamma_{b}$ are involved,

$$
\begin{aligned}
& \int_{\Omega} \mu_{0} \dot{\vec{h}} \cdot \vec{\psi} d \Omega \\
+ & \int_{\Omega_{b}} \mu_{0} \dot{\vec{H}}_{a} \cdot \vec{\psi} d \Omega+\int_{\Gamma_{b}}\left(\mu_{0} \dot{\vec{H}}_{a} \varphi\right) \cdot \vec{n}_{b} d \Gamma \\
+ & \int_{\Omega_{c}} \rho(|\vec{\nabla} \times \vec{h}|)(\vec{\nabla} \times \vec{h}) \cdot(\vec{\nabla} \times \vec{\psi}) d \Omega=0,
\end{aligned}
$$

thereby avoiding the need to describe the asymptotics of the applied field at large distances. Here, $\Omega_{b}$ is an arbitrary box which encloses $\Omega_{c}$, while $\vec{n}_{b}$ stands for the outer normal of $\Gamma_{b}$, as illustrated in Fig. 2.

The model of Eq. (3) is solved with a constitutive relation between the electric field, $\vec{E}$, and the current density, $\vec{j}=$ $\vec{\nabla} \times \vec{h}$, assuming the power law $|\vec{E}|=E_{c}\left(|\vec{j}| / j_{c}\right)^{n}$, from which the electrical resistivity $\rho(|\vec{j}|)=E_{c} / j_{c}\left(|\vec{j}| / j_{c}\right)^{n-1}$ is obtained. Here, $E_{c}=10^{-4} \mathrm{~V} / \mathrm{m}$ is the critical electric field, $n=19$, and $j_{c}$ is the critical current density. Below, $j_{c}$ is either assumed to be constant, or to follow the extended Kim's law with

$$
j_{c}(B)=\frac{j_{c 0}}{\left(1+|\vec{B}| / B_{0}\right)^{\alpha}},
$$

where $\vec{B}=\mu_{0} \vec{H}$ is the magnetic flux density and $\alpha$ is a dimensionless exponent.

The system is composed of a square film of size $200 \times$ $200 \mu \mathrm{m}^{2}$ and a superposed strip of size $200 \times 100 \mu \mathrm{m}^{2}$, with its long edges at equal distances from the edges of the square. In accordance with [22], the parameters which define the geometry of the shell-transformation, $A$ and $B$, are set to $22 \mu \mathrm{m}$ and $44 \mu \mathrm{m}$, respectively. The thickness of each film is $d=300 \mathrm{~nm}$; they are separated by an insulating layer of thickness $t=300 \mathrm{~nm}$. Following [22] the high aspect ratio of the films allows us to treat them as thin films, with one element across the thickness. The insulating layer also contains one element across the thickness. Later, more elements are added when the thickness $t$ is increased in the study reported in part $\mathrm{C}$ of Section $\mathrm{V}$. The magnetic field $\vec{H}_{a}$ is applied

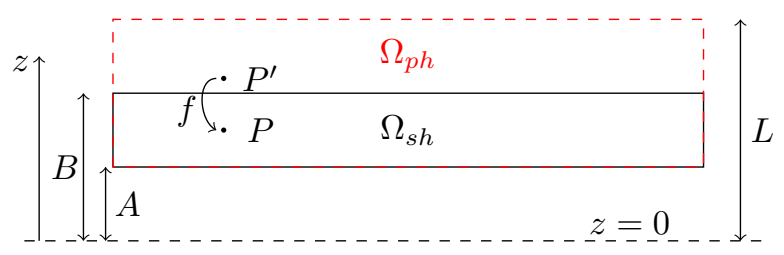

Fig. 3. Illustration of unidirectional shell-transformation along the $\hat{z}$ axis. Each point $P^{\prime}$ in $\Omega_{p h}$ is mapped onto a point $P$ in $\Omega_{s h}$, by means of a change of coordinates, $z=f(Z)$.

perpendicular to the assembly according to a ZFC procedure. It is first ramped up at a constant rate $\dot{H}_{a}=1000 \mathrm{~A} / \mathrm{m}$.s (or $\sim 12.6 \mathrm{Oe} / \mathrm{s}$ ) and then ramped back to 0 at the same rate. The simulations were performed on a Intel Core i7-4790 CPU, $3.6 \mathrm{GHz}, 16 \mathrm{~Gb}$ RAM with an open-source software suite including Gmsh [23] for the mesh generation and GetDp [24] for the finite element solution.

\section{RESULTS}

\section{A. Experimental results}

Figure 4, left-column, shows the distributions of the out-ofplane component of the magnetic field as revealed by MOI after the assembly has been cooled in zero-field and then subjected to an applied field of fixed maximum strength. At the lowest strength of $50 \mathrm{Oe}$, the magnetic flux penetrates the square film through its edges, with discontinuity lines arising along the diagonals and forming an "X" pattern [18]. With increasing maximum strength, the magnetic flux progressively penetrates the central region, where both superconducting layers overlap, and generates new discontinuity lines. A first set of d-lines develops along the bisectors of each of the corners of the central rectangle, initiating the "double Y" pattern usually observed in rectangular thin films [18]. A second set is observed along the edges of the overlapping region, where discontinuity lines are forming as extensions of the legs of the outer " $\mathrm{X}$ " pattern. These inner lines are, however, following directions that are clearly different from the diagonals, as evidenced by the kinks formed near the edges of the overlapping region. As the magnetic flux progresses further in, the inner d-lines converge to the central region of the assembly with an angle which changes with the applied field. For an applied field of 100 Oe, the angle of the inner dlines relative to the vertical direction is seen to be larger than $\pi / 4$. As the applied field increases, this angle decreases and the d-lines tend to align with the diagonals of the square. For an applied field of $500 \mathrm{Oe}$, which shows a nearly complete penetration, it can be observed that the alignment does not appear to be complete. Within the resolution of the MOI image, the inner d-lines do not seem to converge to a single point (the center of the assembly), but they appear to converge instead pair-by-pair on two separate points located above and below the center.

Fig. 5, left-column, shows the magnetic field distributions when the applied field is ramped up to 1000 Oe and then ramped down to a fixed value, after a zero-field-cooled procedure. Most of the d-line structure is similar to that observed 

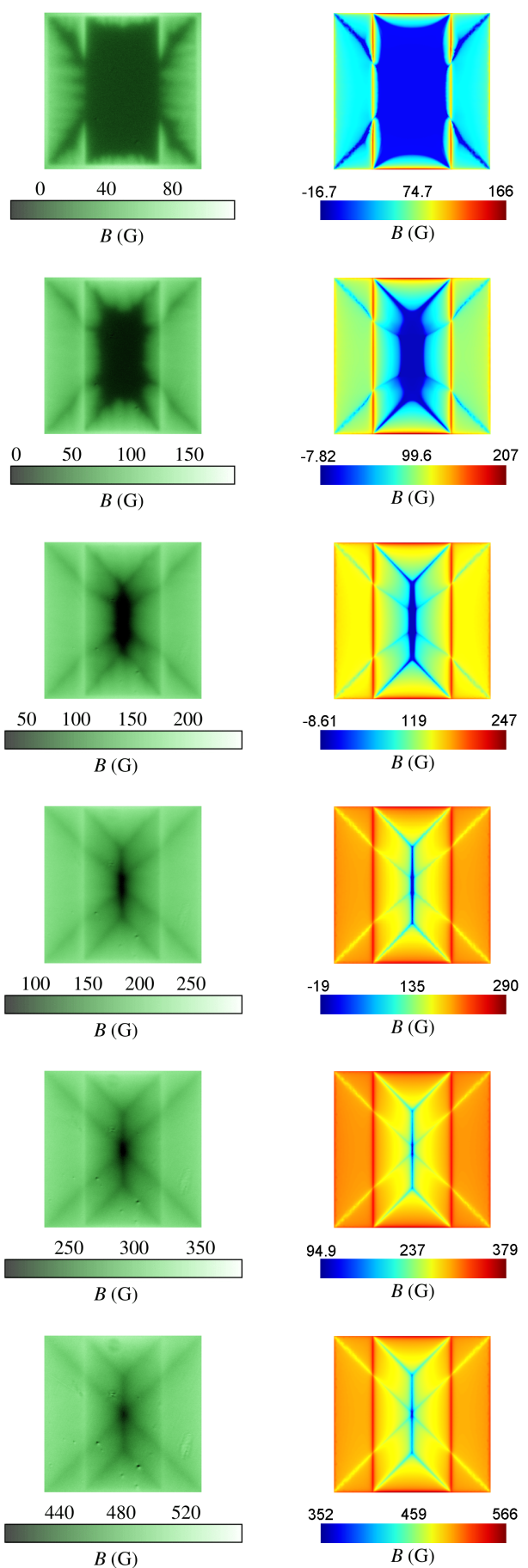

Fig. 4. Distribution of magnetic field in the $\mathrm{Nb}$ bi-layer assembly, where after a zero-field cooling to $6 \mathrm{~K}$, the applied field is ramped up to a fixed value given as, from top to bottom, 50, 100, 150, 200, 300, and $500 \mathrm{Oe}$, respectively. Left column: magneto-optical images showing the experimental distributions of the out-of-plane component of the magnetic field, $B_{z}$ (dark pixels correspond to low field strengths). Right column: simulated distribution of $\mu_{0} H_{z}$.

in Fig. 4: the square film exhibits an " $X$ " pattern, while the overlapping region exhibits the "double Y" pattern. New patterns can, however, be observed in the central part of the overlapping region. The inner d-lines which arise as extension of the external " $\mathrm{X}$ " pattern are now extending toward the central region with an angle relative to the vertical direction
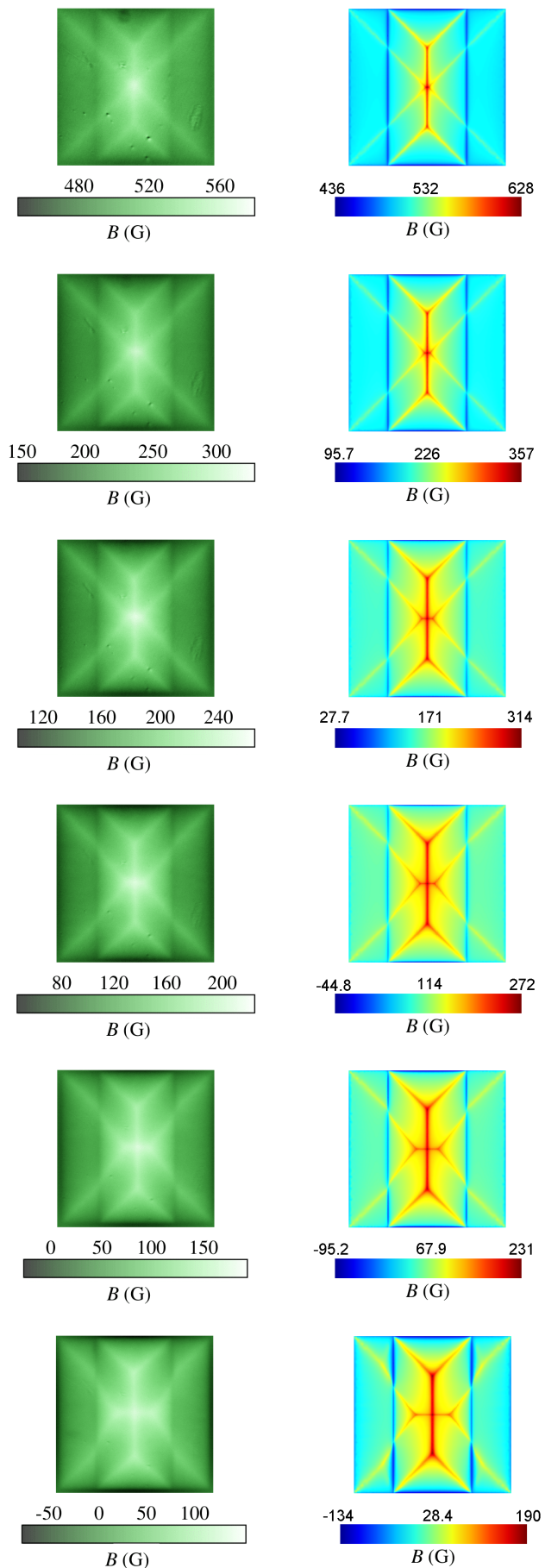

Fig. 5. Distribution of the magnetic field in the $\mathrm{Nb}$ bi-layer assembly, where after a zero-field cooling to $6 \mathrm{~K}$, the applied field is ramped up to 1000 Oe and decreased to a fixed value given as, from top to bottom, 500, 200, 150, 100, 50, and 0 Oe, respectively. Left column: magneto-optical images showing the experimental distributions of $B_{z}$ (dark pixels correspond to low field strengths). Right column: simulated distribution of $\mu_{0} H_{z}$.

which is smaller than $\pi / 4$ and decreases as the final value of the applied field is decreased. Now, the d-lines converge pair-by-pair to two points located left and right of the center. These points are joined by a horizontal segment which forms the new d-line which was first reported in [12]. The length of this new line increases as the final applied field is decreased 
and reaches a maximum extension in the remanent state.

\section{B. Simulation results}

Numerical simulations of the magnetic response were carried out for the same conditions as those which prevailed in experiments. The model of Sec. III was applied assuming an extended Kim's law with different sets of parameters for each film. These parameters were chosen to fit at best the experiments, which are shown in the left column of Fig. 4 and Fig. 5. In the square film, $B_{0}=1.25 \mathrm{mT}, j_{c 0}=3.4$ $\mathrm{MA} / \mathrm{cm}^{2}$ and $\alpha=0.42$, while in the strip, $B_{0}=4.9 \mathrm{mT}$, $j_{c 0}=5.4 \mathrm{MA} / \mathrm{cm}^{2}$ and $\alpha=0.51$. Results are summarized in the right column of Fig. 4 for raising fields applied to a virgin state, and in the right column of Fig. 5 for decreasing fields after an initial magnetization. The main characteristics of the experimental d-line pattern are faithfully reproduced, including the " $\mathrm{X}$ " and double " $\mathrm{Y}$ " patterns, the kinks at the edge of the overlapping region, and the occurrence of a new horizontal d-line in the remanent state.

\section{DISCUSSION}

A. The essential role played by the dependence of $j_{c}$ with the magnetic field
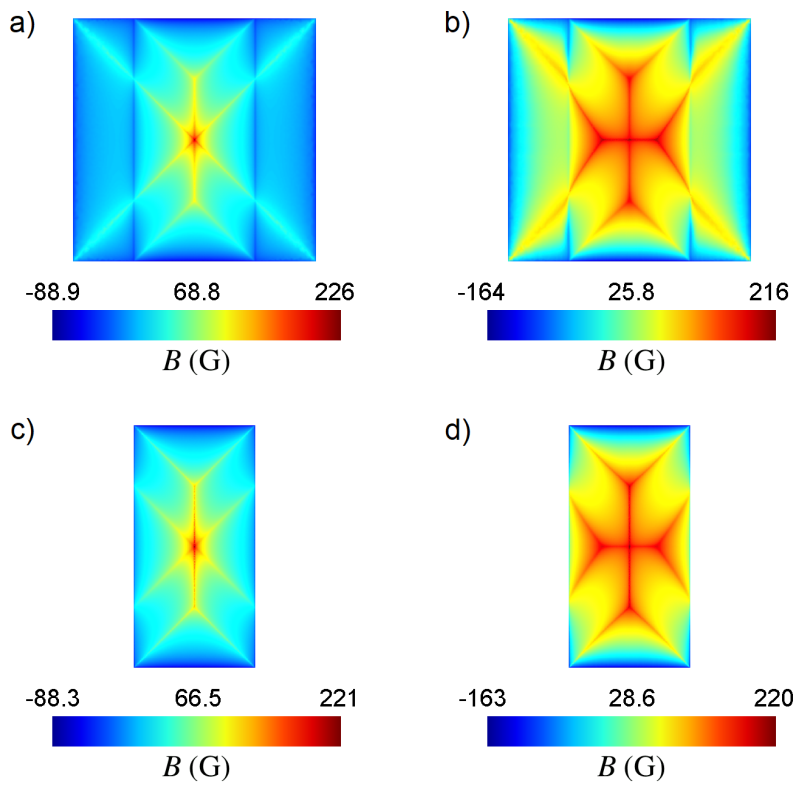

Fig. 6. Simulated $\mu_{0} H_{z}$ distribution for a remanent state in the square film (upper row) and the rectangular strip (lower row), when $j_{c}$ is assumed constant (left column) and when $j_{c}$ is assumed to depend on $|\vec{B}|$ according to Kim's law (right column).

In the following section, we discuss different aspects of the magnetic coupling between the two films, while assuming the same $j_{c}(B)$ laws for each layer, with $\alpha=1$. These sets of parameters simplify somewhat the discussion, while we believe they capture the essential physical mechanisms at play. As we now discuss, assuming an explicit field dependence of the critical current density turns out to be essential in order to reproduce the d-lines observed experimentally. Indeed, this becomes apparent in Figure 6, which shows a comparison between the predictions of the numerical model in two cases: (a), (c), for $j_{c}=2 \mathrm{MA} / \mathrm{cm}^{2}$ assumed constant, and (b), (d), for $j_{c}$ following Kim's law in Eq. (4) with $j_{c 0}=12 \mathrm{MA} / \mathrm{cm}^{2}$, $B_{0}=5 \mathrm{mT}$ and $\alpha=1$ in both films. In each case, the applied field is ramped up to 500 Oe and then decreased to zero to reach a critical state.

The d-lines patterns are strikingly different. For a constant $j_{c}$, the obtained pattern is a mere combination of those expected for a square and rectangular films considered alone: one can identify the " $X$ " pattern running along the diagonals of the square film, with d-lines oriented at an angle $\pi / 4$ relative to the vertical direction, and the "double Y" pattern expected in rectangular films. More specifically, no kink is observed at the edge of the overlapping region. By contrast, the patterns observed experimentally are fully reproduced when $j_{c}(B)$ is assumed to vary with $|\vec{B}|$, as already discussed in Figs. 4 and 5.

It is important to note that essentially the same magnetic flux distribution is seen in both the square and rectangular films, even though they are separated by the electrically insulating layer. As shown in Fig. 6, the distribution of the out-of-plane component of the magnetic flux density are nearly identical. Hence, a common magnetic flux is threading both layers and the same d-lines appear in each layer of the assembly. Experimentally, the magneto-optical images are measuring the magnetic flux which emerges out of the upper part of the assembly and thus also show the same d-lines.


Fig. 7. Distribution of $|\vec{j}|$ and vector map of $\vec{j}$ in the square film (upper row) and in the strip (lower row) for the case of a magnetic field dependent $j_{c}$ and a remanent state (magnetization to 1000 Oe, see text).

Further insight into the magnetic coupling between the two layers can be gained by studying the current distribution in each layer, shown in Fig. 7. It can be observed that even though the two layers share the same distribution of the out-ofplane component of magnetic flux, their current distributions are different. The difference stems from dissimilar in-plane magnetic field components, in accordance with Ampere's law. 




Fig. 8. Critical state in the simplified model corresponding to a magnetization of the square + strip assembly, assuming $J_{c-\text { int }}=2 J_{c-\text { ext }}$ and $W=L / 2$, where $W$ is the width of the strip. Only the upper right part of the structure is shown, the other parts can be obtained by reflection symmetry. Blue lines are d-lines, while thin red lines show the current lines in the square film

The currents in the rectangular film have their flow organized in four sectors, delimited by the "double Y" network of d-lines. In this sense, the current lines are nearly similar to those found in an uncoupled rectangular strip, even though they undergo small deviations near the d-lines originating from the exterior " $X$ " pattern. By contrast, the current lines in the square film are quite different from those expected in an uncoupled film. The current lines are organized in four sectors, delimited by the "X" pattern and its extension in the central region. The most remarkable property is a cell of current rotating around the central horizontal d-line with sharp changes of direction near the lateral edges of the overlapping region, at an angle different from $\pi / 2$. In the overlap region near the upper and lower edges, current lines are subjected to small deviations in the vicinity of the "double Y" network of d-lines.

\section{B. A simplified model}

The current distributions in the remanent state, exhibiting kinks at the edges of the overlapping region, are reminiscent of those observed in a system with an inhomogeneous distribution of the sheet current density $J_{c}(x, y)=\int_{-d / 2}^{d / 2} j_{c}(x, y, z) d z$, see e.g. [13]. In order to focus on the main elements of the magnetic coupling between the two films, we formulate the following simplified planar model: the assembly is divided into a central overlapping region of width $L / 2$ with a constant sheet current density $J_{c \text {-int }}$, while the non-overlapping region (containing two symmetrical parts of width $L / 4$ ) has a constant sheet current density $J_{c-\text { ext }}$. Further, it is assumed that

- upon magnetization from a virgin state, $J_{c-\text { int }}$ and $J_{c-\text { ext }}$ have fixed values with $J_{c-\text { int }}>J_{c-\text { ext }}$, since the magnetic flux is penetrating the non-overlapping region earlier than the overlapping one;

- upon a reduction of the external field after an initial magnetization, $J_{c-\text { int }}$ and $J_{c-\text { ext }}$ have fixed values with

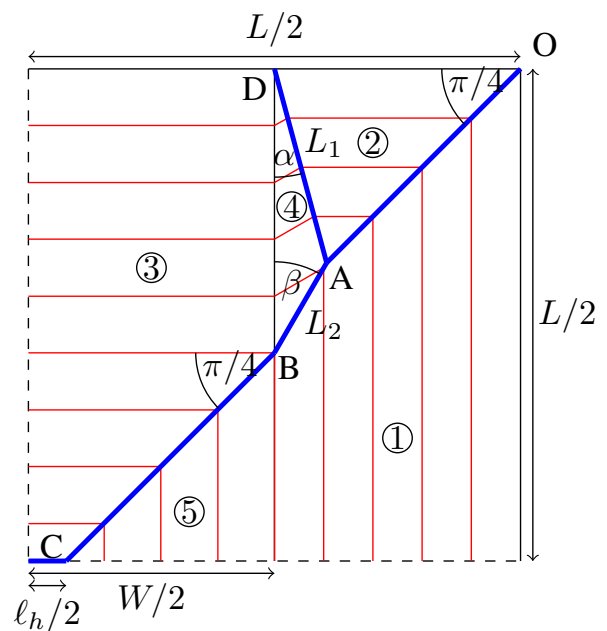

Fig. 9. Critical state in the simplified model corresponding to a remanent state in the square + strip assembly, assuming $J_{c-\text { int }}=\sqrt{3} / 2 \times J_{c-\text { ext }}$ and $W=L / 2$, where $W$ is the width of the strip. Only the upper right part of the structure is shown, the other parts can be obtained by reflection symmetry. Blue lines are d-lines, while thin red lines show the current lines in the square film.

now $J_{c-\text { int }}<J_{c-\text { ext }}$, since the trapped field is on average larger in the overlapping region.

The resulting model is very simplified in the sense that it is two-dimensional and the spatial variations of current densities are neglected within the overlapping and the exterior regions, while only the difference of magnetic field levels between these two regions is taken into account. This model has the advantage that the corresponding critical states can be calculated analytically, yielding Fig. 8 for a magnetization from the virgin state, and Fig. 9 for the remanent state. Quite clearly, the obtained d-lines have a similar structure to that observed in Fig. 4 for the fully magnetized structure, and that found in Fig. 5 for the remanent state. Note in particular the kink appearing along the segments $\mathrm{AD}$ in Fig. 8 and DB in Fig. 9, marking the crossing of current lines between two regions with different sheet current densities.

Of particular interest is the occurence of a vertical d-line of extension $\ell_{v}$ in Fig. 8 and an horizontal line of extension $\ell_{h}$ in Fig. 9. As explained in detail in the appendix, an analysis of the critical states yields

$$
\ell_{v, h}=\frac{L}{2}\left|\frac{J_{c-\mathrm{ext}}}{J_{c-\mathrm{int}}}-1\right|,
$$

which shows that $\ell_{v, h}$ are sensitive to variations of critical currents, induced by spatial variations of magnetic flux densities. To proceed with the analysis, if the average levels of magnetic flux densities are denoted by $\left|\vec{B}_{\text {int }}\right|$ and $\left|\vec{B}_{\text {ext }}\right|$, upon using Kim's law in Eq. (4), with $\alpha=1$, the simplified model predicts that the length of the horizontal line in the remanent state varies as

$$
\ell_{h}=\frac{L}{2} \frac{\left|\vec{B}_{\text {int }}\right|-\left|\vec{B}_{\text {ext }}\right|}{B_{0}+\left|\vec{B}_{\text {ext }}\right|} .
$$

Such a result is not expected to hold strictly in the full threedimensional numerical model, as the magnetic field varies 




Fig. 10. $l_{h} / L$ as a function of $B_{0} / \mu_{0} j_{c 0} d$. The plain red curve is a fit on the data according to Eq. (7) with $C_{1}=0.33$ and $C_{2}=1.44$. The data result from numerical simulations.

spatially and so does the critical current density. Equation 6, however, suggests the possibility to fit the simulated $\ell_{h}$ with the relationship

$$
\frac{\ell_{h}}{L}=\frac{C_{1}}{B_{0} /\left(\mu_{0} j_{c 0} d\right)+C_{2}},
$$

where $C_{1}$ and $C_{2}$ are constants and the product $\mu_{0} j_{c 0} d$ has been chosen as representing a typical level for the magnetic response of the assembly. Figure 10 shows the length of the horizontal line in a series of simulations, where the parameters of Kim's law, $j_{c 0}$ and $B_{0}$, have been varied, together with the fit suggested in Eq. (7). Remarkably, the simulation results fall along a unique curve which is well fitted by Eq. (7). This figure also shows that the horizontal critical line arises when $j_{c}(B)$ varies sufficiently over the range of involved magnetic flux densities. More specifically, $\ell_{h}$ is larger for smaller $B_{0}$, which induce sharper variations of $j_{c}(B)$.

We emphasized the key role played by the $j_{c}(B)$ dependence, leading to an inhomogeneous critical current density distribution. In fact, inhomogeneous critical current densities can also be obtained when an anisotropy is induced by an in-plane magnetic field component [25]. However, note that the strength of in-plane fields which are required to induce a critical current anisotropy in the $\mathrm{Nb}$ films studied here (of thickness of $300 \mathrm{~nm}$ ) is of the order of 300 Oe [25]. Both the in-plane magnetic fields which are obtained in our simulations (less than $60 \mathrm{Oe}$ ) and the in-plane fields which are applied to suppress the magnetic domain in the magneto-optical indicator ( $\sim 180$ Oe) are below this threshold. In addition, if the applied in-plane field ever induces a critical current anisotropy, its principal axes should be parallel to the diagonals of the square, which is different from our observations. Thus, the anisotropy induced by an in-plane component does not appear to play a role in the observed critical states.

\section{Influence of the strength of the magnetic coupling}

Although the simplified model predicted the correct architecture for the d-lines, the information about the average levels



Fig. 11. $\ell_{h} / L$ as a function of $t / d$. The inset depicts the evolution of the normalized scalar magnetic potential $\phi / \phi_{0}$ above the center of a square film of side $L$, as a function of the dimensionless distance $z / L . \phi_{0}$ is the magnetic potential on the boundary of the film.

$\left|\vec{B}_{\text {int }}\right|$ and $\left|\vec{B}_{\text {ext }}\right|$ must still be deduced from the magnetic coupling between the square and the rectangular films. In the remanent state for instance, the magnetization of the rectangular strip, with its typical "roof-top" distribution of $H_{z}$, helps in increasing $\left|\vec{B}_{\text {int }}\right|$ over $\left|\vec{B}_{\text {ext }}\right|$, so that the magnetic coupling is a key element of the resulting critical state. We now study further the influence of the magnetic coupling between the two films by varying the thickness of the insulating layer, $t$, as illustrated in Fig. 11. The simulations were carried out with $j_{c 0}=4 \mathrm{MA} / \mathrm{cm}^{2}, B_{0}=20 \mathrm{mT}$ and $\alpha=1$ in both films, for remanent states. It can be observed that increasing $t$ leads to a shorter $\ell_{h}$, as less magnetic flux emanating from the square film will thread the rectangular film, and vice-versa. The magnetic coupling is monotonically decreasing with $t$, first fairly slowly for $t<10 \mu \mathrm{m}$, and then much more rapidly for larger separations. This change of behavior is reminiscent of the evolution of the magnetic scalar potential $\phi$ along the out-of-plane direction for a superconducting square film, as shown in the inset of Fig. 11. It can be seen that $\phi$ starts decreasing rapidly at a distance $L^{*} \sim 0.1 \times L=20 \mu \mathrm{m}$ from the square film, and so does the magnetic coupling between the two films.

\section{Influence of the width of the rectangular strip}

Last, we investigate the effect of the width, $W$, of the rectangular strip, relative to the side of the square film, $L$. Figure 12 shows the d-lines obtained in a remanent state under the same conditions as those of Sec. IV, for rectangular strips of varying width, with $W / L=1 / 6,1 / 3,1 / 2$, and $2 / 3$. It can be observed that the horizontal d-line for $W / L=2 / 3$ is shorter than that for $W / L=1 / 2$. This reduction can be explained simply, as $\ell_{h}$ is expected to vanish in the limit $W / L=1$, for a stack of two squares. Another way to see this is to observe that an increase of the area of the overlap region leads to a reduction of the average field $\left|\vec{B}_{\text {int }}\right|$ and thus of the ratio of critical current densities $J_{c-\text { ext }} / J_{c-\text { int }}$, hence a smaller $\ell_{h}$. For small $W / L$, it is observed that $\ell_{h}$ increases slightly from $W / L=1 / 2$ to $W / L=1 / 3$, and 
a)



c)

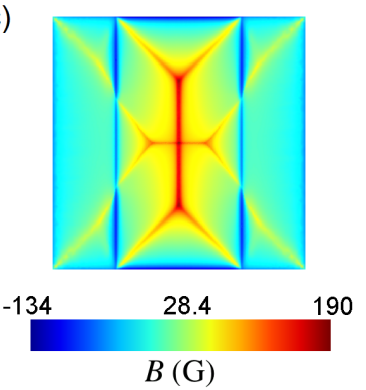

b)

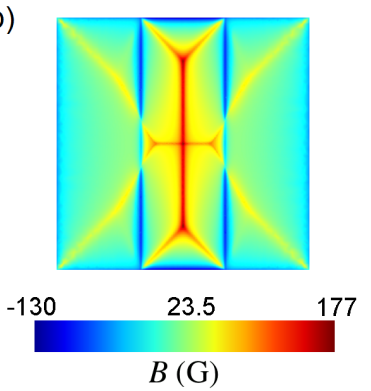

d)

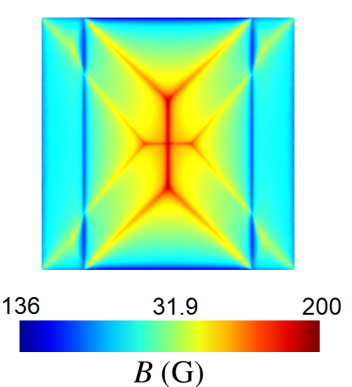

Fig. 12. Distribution of $\mu_{0} H_{z}$ in the square film in the remanent state for different values of the strip width, $W$. a) $W=L / 6$, b) $W=L / 3$, c) $W=L / 2$ and d) $W=2 L / 3$. The parameters of the extended Kim's law are the same as in Sec. IV.

becomes roughly equal to $W$ for $W / L=1 / 6$. To sum up, $\ell_{h}$ will increase with $\sim W$ for small $W / L$, reach a maximum at intermediate $W / L$, and decrease again as $W \rightarrow L$.

\section{CONCLusions}

In this work, we investigated numerically the critical states in a bi-layered structure made of a thin superconducting square film and a rectangular superconducting strip. We showed that the inhomogeneous distribution of critical current density in the films, which is induced by the magnetic field dependence of the critical current density and the spatial distribution of $\vec{B}$, plays a crucial role in the geometry of the critical state. We studied the evolution of the magnetic response of the assembly under a varying applied magnetic field and showed that some d-lines evolve with magnetic field. We suggested a simplified model which reproduces the variations of the new horizontal d-line observed in the remanent state as a function of the parameters $j_{c 0}$ and $B_{0}$. Last, we showed that the magnetic coupling driving the observed patterns persists up to separations between the two superconducting films of about $10 \%$ of the lateral size of the films. The findings on this simple assembly are expected to contribute in understanding how the critical state is built in more complex three-dimensional assemblies of planar superconducting components. Several findings on this simple assembly can be used to predict the critical states in more complex three-dimensional assemblies such as stacks of strips or tapes. We can anticipate that the magnetic coupling will be most important for nearby layers, placed at a distance of less than a few tenths of their lateral sizes. Moreover, the effects will be more pronounced when these layers have different characteristic sizes. Although we investigated only the effect of the lateral sizes, it will be

interesting to study the effects of different parameters, such as the thickness of the films, their relative positions and orientations, and the variations of their shapes.

\section{APPENDIX}

We derive here the expressions for the extensions of the central discontinuity lines, $\ell_{v}$ and $\ell_{h}$, in the simplified model with an inhomogeneous distribution of sheet current densities.

\section{A. Case where $J_{c}$ is larger in the overlapping region}

Assume $W=L / 2$ and $J_{c-\text { int }}>J_{c-\text { ext }}$, yielding the critical state shown in Fig. 8. Consider the segment AD, separating region 2 and region 4 , with respective sheet current densities $J_{c-\text { ext }}$ and $J_{c-\text { int }}$. According to Schuster [13], the horizontal current lines originating from region 2 are deflected an angle $2 \alpha$ to the right upon entering region 4 , with

$$
\frac{J_{c-\text { int }}}{J_{\text {c- ext }}}=\frac{1}{\cos 2 \alpha} \text {. }
$$

The vertical current lines originating from region 5 are deflected into region 4 along $L_{2}$, while keeping the same sheet current density $J_{c-\text { int }}$. Hence, they are deflected an angle $\pi-2 \beta$ to the left. As current lines in region 5 are perpendicular to those in region 2 , one has

$$
-(\pi-2 \beta)=-\pi / 2+2 \alpha \Longrightarrow \beta=\alpha+\frac{\pi}{4} .
$$

In order to relate the extension $\ell_{v}$ to angles $\alpha$ and $\beta$, take the projections of the path OABC along the horizontal and the vertical directions, and calculate their difference. This procedure gives

$$
\ell_{v}=2 L_{2}(\sin \beta-\cos \beta) .
$$

Then, solving the triangle $\mathrm{ABD}$ leads to

$$
L_{2}=\frac{L}{4} \frac{\sin \alpha}{\sin (\pi+\alpha-\beta)} .
$$

Collecting Eqs. (8) to (11) finally gives

$$
\frac{\ell_{v}}{L}=\frac{1-\cos 2 \alpha}{2}=\frac{1}{2}\left(1-\frac{J_{c-\mathrm{ext}}}{J_{c-\mathrm{int}}}\right) .
$$

Note that in the extreme case $\alpha=\pi / 4$, B is located on the vertical median of the square, in such a way that $C$ is the same point as B. Moreover, regions 4 and 5 merge together, so that the segment $\mathrm{AB}$ becomes horizontal and is not considered as a d-line any more.

\section{B. Case where $J_{c}$ is larger in the peripheral region}

We now turn to the case where $J_{c-\text { ext }}>J_{c \text {-int }}$, using similar arguments. Again, all calculations are made assuming $W=L / 2$. The corresponding critical state is shown in Fig. 9 . Consider first the current lines originating from region 2 . These are deflected into region 4 by an angle $2 \alpha$ to the left, with, following Schuster [13],

$$
\frac{J_{c-\text { ext }}}{J_{\text {c-int }}}=\frac{1}{\cos 2 \alpha} \text {. }
$$


Similarly, current lines originating from region 1 and entering region 4 through the segment $\mathrm{AB}$ are deflected an angle $\pi-2 \beta$ to the left. Since these current lines are perpendicular to those entering region 4 through the segment $\mathrm{AD}$, one has

$$
-(\pi-2 \beta)=-\pi / 2-2 \alpha \Longrightarrow \beta=\frac{\pi}{4}-\alpha .
$$

The length $\ell_{h}$ is again obtained by evaluating the difference between the projections of the path OABC on the vertical and horizontal directions. This procedures yields

$$
\ell_{h}=2 L_{2}(\cos \beta-\sin \beta) .
$$

Next, solve successively triangles OAD and DAB to obtain

$$
L_{1}=\frac{L}{4} \frac{\sin \pi / 4}{\sin (3 \pi / 4-\alpha)},
$$

and

$$
L_{2}=L_{1} \frac{\sin \alpha}{\sin \beta} .
$$

Collecting Eqs. (13) to (17), we finally have

$$
\frac{\ell_{h}}{L}=\frac{1}{2}\left(\frac{1}{\cos 2 \alpha}-1\right)=\frac{1}{2}\left(\frac{J_{c-\mathrm{ext}}}{J_{c-\mathrm{int}}}-1\right) .
$$

Similarly to the previous case, the d-lines depicted in Fig. 9 are only valid if

$$
\frac{1}{2}<\cos 2 \alpha
$$

Otherwise, the segment $\mathrm{AB}$ does not intersect the vertical line which delimits the two regions of different critical current densities. The segment $\mathrm{AB}$ slides down and crosses the median line at some new point. As a result, region 5 is not apparent, and Eq. 18 is invalidated.

\section{REFERENCES}

[1] Eds. V. Moshchalkov, R. Wordenweber and W. Lang 2010 Nanoscience and Engineering in Superconductivity (Springer Berlin Heidelberg) (https://www.springer.com/kr/book/9783642151361)

[2] D. Rosenberg et al. 2018 3D integrated superconducting qubits $n p j$ Quantum Information 342

[3] E. E. Mitchell and C.P. Foley 2010 YBCO step-edge junctions with high IcRn Superconductor Science and Technology 23065007

[4] E. J. Romans, E. J. Osley, L. Young, P. A. Warburton and W. Li 2010 Three-dimensional nanoscale superconducting quantum interference device pickup loops Applied Physics Letters 97222506

[5] C. Navau, J. Prat-Camps and A. Sanchez 2012 Magnetic energy harvesting and concentration at a distance by transformation optics Physical Review Letters 109263903

[6] F. Gömöry, M. Solovyov, J. Šouc, C. Navau, J. Prat-Camps and A. Sanchez 2012 Experimental Realization of a Magnetic Cloak Science 335 1466-1468

[7] M. Nii, N. Amemiya and T. Nakamura 2012 Three-dimensional model for numerical electromagnetic field analyses of coated superconductors and its application to Roebel cables Superconductor Science and Technology 25119501

[8] A. Patel, A. Baskys, T. Mitchell-Williams, A. McCaul, W. Coniglio, J. Hänish, M. Lao and B. A. Glowacki 2018 A trapped field of 17.7 T in a stack of high temperature superconducting tape Superconductor Science and Technology 31 09LT01

[9] L. Prigozhin and V. Sokolovsky 2018 Solution of 3D magnetization problems for superconducting film stacks Superconductor Science and Technology 31125001
[10] S. Hahn, K. Kim, K. Kim, X. Hu, T. Painter, I. Dixon, S. Kim, K. R. Bhattarai, S. Noguchi, J. Jaroszynski and D. C. Larbalestier 2019 45.5tesla direct-current magnetic field generated with a high-temperature superconducting magnet Nature 570 496-499

[11] T. Tamegai, Y. Tsuchiya, S. Tada, J. Ibuka, A. Mine, S. Pyon, Y. Mawatari, S. Nagasawa, M. Hikada and M. Maezawa 2014 Flux penetrations into two- and three-dimensional nanostructured superconductors Physica C $\mathbf{5 0 3}$ 62-69

[12] T. Tamegai, A. Mine, Y. Tsuchiya, S. Miyano, S. Pyon, Y. Mawatari, S. Nagasawa and M. Hidaka 2017 Critical states and thermomagnetic instabilities in three-dimensional nanostructured superconductors Physica C 533 74-79

[13] Th. Schuster, M. V. Indenbom and H. Kuhn 1995 Discontinuity lines in rectangular superconductors with intrinsic and extrinsic anisotropies Physical Review B 5215621

[14] Th. Schuster, H. Kuhn and E. H. Brandt 1996 Flux penetration into flat superconductors of arbitrary shape: Patterns of magnetic and electric fields and current Physical Review B 54 3514-3524

[15] J. I. Vestgarden, D. V. Shantsev, Y. M. Galperin and T. H. Johansen 2008 Flux distribution in superconducting films with holes Physical Review B 77014521

[16] J. Brisbois, O.-A. Adami, J. Avila Osses, M. Motta, W. A. Ortiz, N. D. Nguyen, P. Vanderbemden, B. Vanderheyden, R. B. G. Kramer and A. V. Silhanek 2016 Magnetic flux penetration in $\mathrm{Nb}$ superconducting films with lithographically defined micro-indentations Physical Review B 93054521

[17] K.A. Lorincz, M.S. Welling, J.H. Rector and R.J. Wijngaarden 2004 Flux penetration in patterned superconducting thin films with multiply connected geometry Physica $C \mathbf{4 1 1} 1-10$

[18] Th. Schuster, H. Kuhn, S. Klaumünzer and E. H. Brandt 1997 Flux penetration into flat rectangular superconductors with anisotropic critical current Physical Review B 56 3413-3424

[19] Th. Schuster, H. Kuhn, E. H. Brandt, M. V. Indenbom, M. Kläser, G. Müller-Vogt, H.-U. Habermeier, H. Kronmüller and A. Forkl 1995 Current and field pattern in rectangular and inhomogeneous superconductors Physical Review B 52 10375-10389

[20] S. Nagasawa, K. Hinode, T. Satoh, H. Akaike, Y. Kitagawa and M. Hidaka 2004 Development of advanced $\mathrm{Nb}$ process for SFQ circuits Physica C 412-414 1429-1436

[21] Y. Tsuchiya, Y. Mawatari, J. Ibuka, S. Tada, S. Pyon, S. Nagasawa, M. Hidaka, M. Maezawa, and T. Tamegai 2013 Flux Avalanches in Nb Superconducting Shifted Strip Arrays Superconductor Science and Technology 26095004

[22] L. Burger, C. Geuzaine, F. Henrotte and B. Vanderheyden 2019 Modelling the penetration of magnetic flux in thin superconducting films with shell transformations International Journal for Computation and Mathematics in Electrical and Electronic Engineering Vol. ahead-ofprint No. ahead-of-print

[23] C. Geuzaine and J.-F. Remacle 2009 Gmsh: a three-dimensional finite element mesh generator with built-in pre- and post-processing facilities International Journal for Numerical Methods in Engineering 79 13091331

[24] GetDP: A General Environment for the Treatment of Discrete Problems http://getdp.info/

[25] F. Colauto, D. Carmo, A. M. H. de Andrare, A. A. M. Oliveira, W. A. Ortiz, Y. M. Galperin and T. H. Johansen 2019 Anisotropic flux penetration in superconducting $\mathrm{Nb}$ films with frozen-in in-plane magnetic fields IEEE Transactions on Applied Superconductivity 29 8002505 